\title{
Discovery of complex narrow $X$-ray absorption features from the low-mass $X$-ray binary GX $13+1$ with XMM-Newton
}

\author{
L. Sidoli ${ }^{1}$, A. N. Parmar ${ }^{2}$, T. Oosterbroek ${ }^{2}$, and D. Lumb ${ }^{2}$ \\ 1 Istituto di Fisica Cosmica "G. Occhialini", CNR, via Bassini 15, 20133 Milano, Italy \\ 2 Astrophysics Division, Research and Scientific Support Department of ESA, ESTEC, Postbus 299, \\ 2200 AG Noordwijk, The Netherlands
}

Received 19 December 2001 / Accepted 31 January 2002

\begin{abstract}
We report the detection of a complex of narrow $\mathrm{X}$-ray absorption features from the low-mass $\mathrm{X}$-ray binary GX 13+1 during 3 XMM-Newton observations in 2000 March and April. The features are consistent with being due to resonant scattering of the $\mathrm{K} \alpha$ and $\mathrm{K} \beta$ lines of He- and H-like iron (Fe XXV and Fe XxVI) and H-like calcium (Ca Xx) K $\alpha$. Only the Fe Xxvi K $\alpha$ line has been previously observed from GX 13+1. Due to the closeness in energy the FeXXV and Fexxvi K $\beta$ features may also be ascribed to Ni XXVII and Ni XXVIII K $\alpha$, respectively. We also find evidence for the presence of a deep $(\tau \sim 0.2)$ Fe XxV absorption edge at $8.83 \mathrm{keV}$. The fits also require the presence of a broad emission feature whose energy and width are poorly determined, partly due to the presence of the deep $\mathrm{Fe} \mathrm{K} \alpha$ features which severely cut into the feature and partly due to fit differences when using different XMM-Newton instruments. The equivalent widths of the lines do not show any obvious variation on a timescale of a few days suggesting that the absorbing material is a stable feature of the system and present during a range of orbital phases.
\end{abstract}

Key words. accretion, accretion disks - stars: individual: GX 13+1 - stars: neutron - X-rays: general

\section{Introduction}

The bright and persistent low-mass $\mathrm{X}$-ray binary (LMXRB) GX 13+1 (4U 1811-17) is an X-ray burst source (Fleischman 1985; Matsuba 1995) with radio (Grindlay \& Seaquist 1986) and infrared counterparts (Naylor et al. 1991; Charles \& Naylor 1992). It displays a $15 \%$ modulation in X-ray intensity with a period of $24.7 \pm 1$ days. The $\mathrm{X}$-ray intensity is anti-correlated with the hardness ratio (Corbet 1996). Recent estimates of the spectral type of the companion (an evolved late-type K5 III star; Bandyopadhyay et al. 1999), together with the binary system parameters are consistent with this modulation being the orbital period, although further confirmation is required (Bandyopadhyay et al. 2002). GX 13+1 has been classified as an atoll source, but in some respects its properties are more similar to those of the Z-sources (Homan et al. 1998; Schulz et al. 1989; Bandyopadhyay et al. 1999), having persistent radio emission at a similar level to the Z-sources (Fender \& Hendry 2000), and a high accretion rate of $\sim 0.1$ Eddington. A quasi-periodic oscillation (QPO) was discovered at $57-69 \mathrm{~Hz}$ by Homan et al. (1998). Distance estimates for GX 13+1 range from 4.7 to

Send offprint requests to: L. Sidoli,

e-mail: sidoli@ifctr.mi.cnr.it
7.0 kpc (Christian \& Swank 1997). Infrared spectroscopy favors a distance of $7 \pm 1 \mathrm{kpc}$ (Bandyopadhyay et al. 1999).

A spectral survey of Fe-K emission from 20 LMXRB with ASCA is reported in Asai et al. (2000). Significant iron emission was detected from GX $13+1$ with a line centroid of $6.44 \pm 0.05 \mathrm{keV}$, an equivalent width $(E W)$ of $37_{-8}^{+10} \mathrm{eV}$ and a line width $\sigma \sim 130 \pm 40 \mathrm{eV}$. The Xray continuum could be modeled with a two-component model, consisting of a multicolor disk-blackbody with a temperature, $k T$, at the inner disk radius of $0.76 \mathrm{keV}$ and a blackbody with a $k T$ of $1.34 \mathrm{keV}$, absorbed by a column density, $N_{\mathrm{H}}$, of $2.9 \times 10^{22}$ atom $\mathrm{cm}^{-2}$. The source flux (1-10 keV, corrected for absorption) was $1.2 \times 10^{-8} \mathrm{erg} \mathrm{cm}^{-2} \mathrm{~s}^{-1}$, which translates into a luminosity of $7 \times 10^{37} \mathrm{erg} \mathrm{s}^{-1}$ (at $7 \mathrm{kpc}$ ). Recently, Ueda et al. (2001) re-analyzed the ASCA Solid-State Imaging Spectrometer (SIS) observation of GX $13+1$ and discovered that the spectral region around Fe is more complex than had been realized. Their fits required the presence of a narrow $(\sigma<70 \mathrm{eV})$ absorption line at $7.01 \pm 0.03 \mathrm{keV}$ with an $E W$ of $35 \pm 8 \mathrm{eV}$, which they interpreted as resonant scattering of the K $\alpha$ line from Fe XxVI ions with a column density $\gtrsim 10^{18} \mathrm{~cm}^{-2}$, a narrow $(\sigma<220 \mathrm{eV})$ emission line at $6.42 \pm 0.08 \mathrm{keV}$ with an $E W$ of $19 \pm 8 \mathrm{eV}$ and an absorption edge at $7.61 \pm 0.13 \mathrm{keV}$ with an 

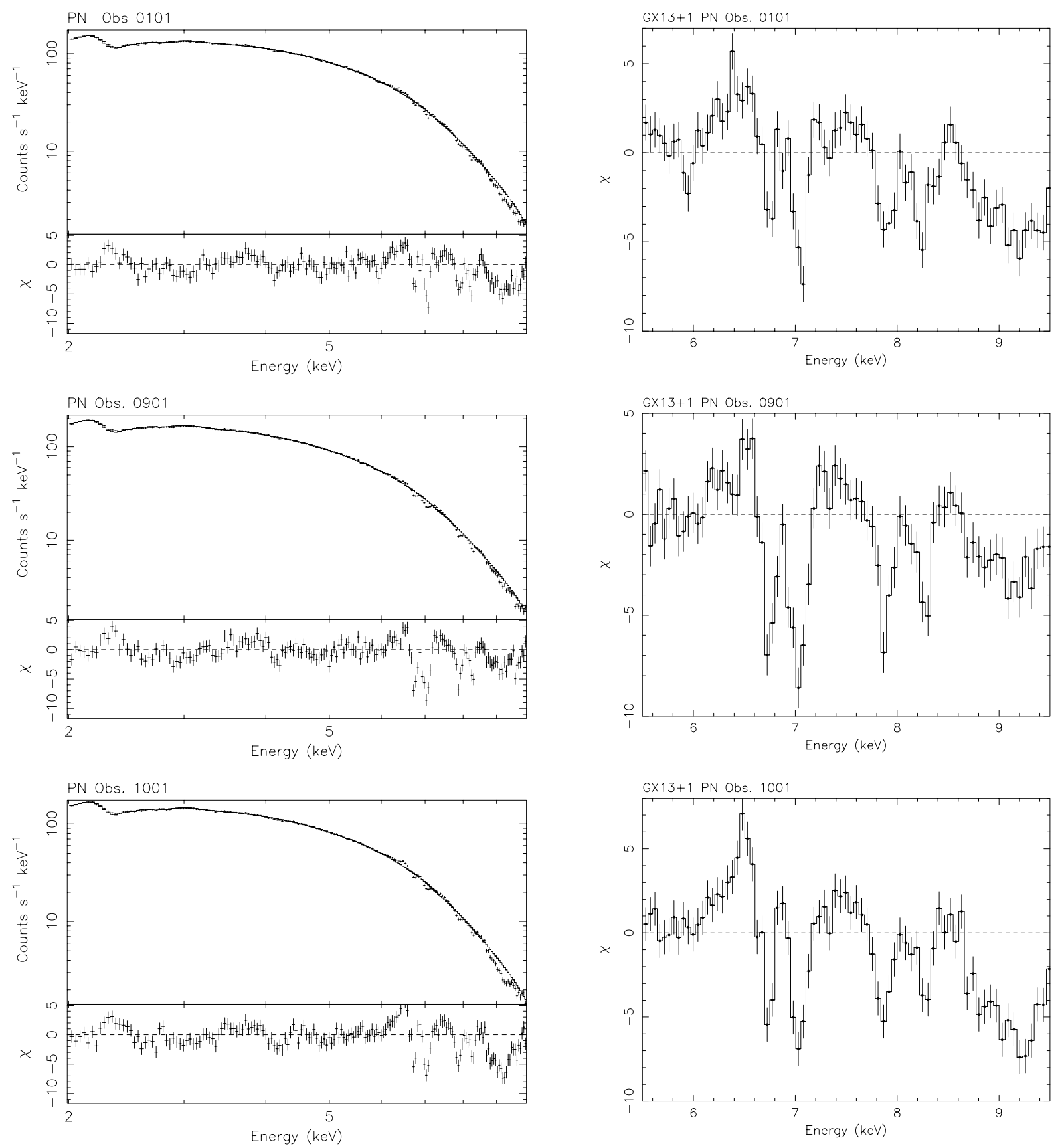

Fig. 1. The EPIC PN spectra for the 3 on-axis observations of GX 13+1. In the left panels, the best-fit continuum model consisting of absorbed multi-color blackbody and blackbody components, together with a broad emission feature at $6.8 \mathrm{keV}$ is shown with the normalizations of the narrow discrete absorption features, and the optical depth of the absorption edge, set to zero. On the right, expanded views of the residuals (in units of standard deviation) in the 5.5-9.5 keV energy range. The four absorption lines, a broad emission line at $6.8 \mathrm{keV}$ which is strongly affected by the two strongest absorption features and the absorption edge at $8.83 \mathrm{keV}$ are clearly visible

optical depth of $0.13 \pm 0.05$. This was the first detection of an Fe absorption line in an X-ray binary known to contain a neutron star. Previously, the only $\mathrm{X}$-ray binaries known to exhibit such Fe absorption features were two superluminal jet sources GRO J1655-40 (Ueda et al. 1998; Yamaoka et al. 2001) and GRS 1915+105 (Kotani et al. 2000; Lee et al. 2001). As Ueda et al. (2001) comment, the interpretation of the edge is not simple and it could be a combination of an absorption edge from moderately ionized Fe together with line absorption from a number of prominent $\mathrm{K} \alpha$ or $\mathrm{K} \beta$ lines of $\mathrm{Fe}$ and $\mathrm{Ni}$.

XMM-Newton is significantly improving our understanding of the LMXRB emission processes, accretion geometries and the physical conditions of the material through which the $\mathrm{X}$-ray emission propagates. Reflection Grating Spectrometer (RGS) observations of the eclipsing and dipping LMXRB EXO 0748-676 (Cottam et al. 2001) revealed several discrete absorption and emission 
Table 1. XMM-Newton on-axis observation log of GX 13+1. All observations used the medium filter.

\begin{tabular}{lrrccl}
\hline \hline Rev. & Obs. ID & $\begin{array}{r}\text { Inst. } \\
\text { EPIC }\end{array}$ & $\begin{array}{r}\text { Start time (2000) } \\
(\text { dy mon hr:mn })\end{array}$ & $\begin{array}{r}\text { Net Exp. } \\
(\mathrm{ks})\end{array}$ & Mode \\
\hline \multirow{2}{*}{056} & 0101 & MOS1 & 30 Mar. 15:13 & 5.9 & Full Frame \\
& & PN & 30 Mar. 14:47 & 2.5 & Timing \\
0057 & \multirow{2}{*}{0901} & MOS1 & 01 Apr. 06:28 & 4.9 & Full Frame \\
& & PN & 01 Apr. 05:52 & 2.1 & Timing \\
0057 & \multirow{2}{*}{1001} & MOS1 & 01 Apr. 09:54 & 4.6 & Full Frame \\
& & PN & 01 Apr. 09:28 & 2.3 & Timing \\
\hline
\end{tabular}

features from ionized neon (Nex Ly $\alpha$, Ne IX Ly $\alpha$ ), oxygen (O viII Ly $\alpha$, O viI He-like complex) and nitrogen (N VII Ly $\alpha$ ). These line features are broadened (velocity widths of $\left.1000-3000 \mathrm{~km} \mathrm{~s}^{-1}\right)$, but with no evidence for a velocity shift $\left(<300 \mathrm{kms}^{-1}\right)$. No obvious dependence on orbital phase was found.

During an XMM-Newton observation of the eclipsing and dipping LMXRB MXB 1659-298 resonant absorption features identified with $O$ VIII $1 s-2 p, 1 s-3 p$ and $1 s-4 p$, Ne x $1 s-2 p$, Fe XXV 1s-2p, and Fe XXVI 1s-2p transitions, together with a broad Fe emission feature at $6.47_{-0.14}^{+0.18} \mathrm{keV}$ were discovered (Sidoli et al. 2001). The line widths are unresolved in the RGS and correspond to velocities of $<600 \mathrm{kms}^{-1}$ for O VIII $1 \mathrm{~s}-2 \mathrm{p}$ and $<2300 \mathrm{kms}^{-1}$ for the Fe XXvi K $\alpha$ line observed using the European Photon Imaging Camera (EPIC). The absorption features occur through a wide range of orbital phases, so the absorbing material is most likely located above, or below, the accretion disk, in a cylindrical geometry with an axis perpendicular to that of the disk.

Similar absorption features have been reported by Parmar et al. (2002) during an XMM-Newton observation of the LMXRB X 1624-490, sometimes called the "Big Dipper". Here the lines are identified with the resonant $\mathrm{K} \alpha$ absorption of Fe XXV and Fe XXVI, with Ni XXVII K $\alpha$ and Fexxvi K $\beta$ possibly detected. Again, no obvious dependence on orbital phase was observed, except during an absorption dip. Here, we report the discovery of discrete $\mathrm{X}$-ray absorption features from highly ionized $\mathrm{Fe}$ and $\mathrm{Ca}$ (and possibly $\mathrm{Ni}$ ) in XMM-Newton EPIC spectra of GX $13+1$.

\section{Observations}

The XMM-Newton Observatory (Jansen et al. 2001) includes three $1500 \mathrm{~cm}^{2} \mathrm{X}$-ray telescopes each with an EPIC at the focus. Two of the EPIC imaging spectrometers use MOS CCDs (Turner et al. 2001) and one uses a PN CCD (Strüder et al. 2001). The region of sky containing GX 13+1 was observed by XMM-Newton a number of times as part of the calibration programme. Three onaxis observations were performed (see Table 1). Since an analysis of two off-axis observations (2000 April 1 13:34 and April 3 13:34 UTC) give similar spectral results, we discuss only the better calibrated on-axis observations. In order to minimize the effects of pile-up the PN and MOS2 were operated in their Timing Modes. The MOS1 CCD was operated in its normal Full Frame Mode and the data are strongly affected by pile-up (when two photons are detected closely in time and the energy is incorrectly assigned to be the sum of the individual energies; see Strüder et al. 2001). The effects of pile-up in MOS1 were minimized by extracting events in an annulus outside the $30^{\prime \prime}$ radius core of the GX $13+1$ point spread function (PSF). Since there are far fewer MOS events than PN ones, the MOS1 data are only used to verify the overall shape and energy calibration of the PN data.

Raw data products were extracted from the public XMM-Newton archive and then reprocessed using version 5.2 of the Science Analysis Software (SAS), before being further filtered using XMMSELECT. Only X-ray events corresponding to patterns 0 (single pixel events) were selected. Background spectra were obtained from source free regions taken from the same observations. For the Timing Mode observations, appropriate public response matrices are not yet available. Thus, the standard PN matrix for Full Frame Mode has been adopted. This slightly affects the modeling of the continuum, and possibly the energies of the lines (F. Haberl, priv. communication), but is unlikely to affect estimates of their $E W \mathrm{~s}$. We concentrate here on the analysis of the narrow absorption features evident in the spectra and defer a detailed discussion of the continuum properties to a later paper.

\section{Results}

A total of 6 EPIC spectra of GX 13+1 (3 MOS1 and 3 PN) from the on-axis observations reported in Table 1 were extracted. These were then rebinned to oversample the $F W H M$ of the energy resolution by a factor 3 and to have additionally a minimum of 20 counts per bin to allow use of the $\chi^{2}$ statistic. In order to account for systematic effects a $2 \%$ uncertainty was added quadratically to each spectral bin. The photo-electric absorption cross sections of Morrison \& McCammon (1983) are used throughout. All spectral uncertainties are given at $90 \%$ confidence, unless indicated otherwise.

The overall 2.0-10 keV continua were investigated using the same model as applied to the ASCA spectrum reported in Ueda et al. (2001) consisting of absorbed blackbody and multicolor disk-blackbody (Mitsuda et al. 1984) components. Fits were made to all 6 spectra individually. 
Table 2. Parameters of the absorption lines detected in the XMM-Newton spectra of GX $13+1$. A continuum model consisting of absorbed blackbody and disk-blackbody components was used.

\begin{tabular}{|c|c|c|c|c|c|c|c|}
\hline \multirow[t]{2}{*}{ Component } & \multirow[t]{2}{*}{ Parameter } & \multicolumn{2}{|c|}{ Obs. 0101} & \multicolumn{2}{|c|}{ Obs. 0901} & \multicolumn{2}{|c|}{ Obs. 1001} \\
\hline & & MOS1 & $\mathrm{PN}$ & MOS1 & $\mathrm{PN}$ & MOS1 & $\mathrm{PN}$ \\
\hline CaX & $\mathrm{reV})$ & $4.15_{-0.05}^{+0.05}$ & 4.15 & $4.16_{-0.03}^{+0.03}$ & $4.18^{+}$ & $4.13_{-0.03}^{+0.04}$ & 4.19 \\
\hline line & & & $<0$ & & $<0$. & & $<0.1$ \\
\hline & $E W(\mathrm{eV})$ & $-7.6_{-7.6}^{+7.1}$ & $-3.3_{-2.1}^{+1.9}$ & $-11.3_{-7.0}^{+4.3}$ & $-4.3_{-2.3}^{+2.1}$ & $-7.7_{-5.8}^{+3.8}$ & $-6.0_{-2.9}^{+2.6}$ \\
\hline Fexxv K $\alpha$ abs & $E_{\text {line }}(\mathrm{keV})$ & $6.73_{-0.04}^{+0.04}$ & $6.75_{-0.02}^{+0.10}$ & $6.76_{-0.05}^{+0.05}$ & $6.75_{-0.02}^{+0.01}$ & $6.72_{-0.06}^{+0.08}$ & $6.72_{-0.01}^{+0.02}$ \\
\hline line & $\sigma(\mathrm{keV})$ & $<0.1$ & $<0.1$ & $<0.1$ & $<0.1$ & $<0.05$ & $<0.04$ \\
\hline & $E W(\mathrm{eV})$ & $-23.2_{-19.2}^{+13.2}$ & $-23.2_{-15.1}^{+4.6}$ & $-26.7_{-17.3}^{+17.3}$ & $-37.5_{-17.0}^{+9.7}$ & $-17.2_{-15.1}^{+13.6}$ & $-32.2_{-8.2}^{+10.1}$ \\
\hline Fexxvi K $\alpha$ abs & $E_{\text {line }}(\mathrm{keV})$ & $7.00_{-0.02}^{+0.02}$ & $7.00_{-0.10}^{+0.03}$ & $7.06_{-0.04}^{+0.04}$ & $7.03_{-0.02}^{+0.01}$ & $7.03_{-0.03}^{+0.07}$ & $7.05_{-0.02}^{+0.02}$ \\
\hline line & $\begin{array}{l}\sigma(\mathrm{keV}) \\
E W(\mathrm{eV})\end{array}$ & $\begin{array}{l}<0.05^{+18.0} \\
-51.3_{-18.5}^{+18}\end{array}$ & $\begin{array}{l}<0.04 \\
-36.1_{-5.6}^{+2.5}\end{array}$ & $\begin{array}{l}<0.05^{+20.9} \\
-57.0_{-22.9}^{+20}\end{array}$ & $\begin{array}{l}<0.05^{+6.5} \\
-56.5_{-14.6}^{+6 .}\end{array}$ & $\begin{array}{l}<0.05^{+19.5} \\
-39.6_{-20.5}^{+19}\end{array}$ & $\begin{array}{l}<0.06 \\
-39.4_{-7.6}^{+6.1}\end{array}$ \\
\hline Fex & $E_{\text {line }}(\mathrm{keV})$ & 7.85 fixed & $7.90_{-0.04}^{+0.01}$ & 7.85 fixed & $7.87_{-0.03}^{+0.05}$ & $7.84_{-0.04}^{+0.04}$ & $7.85_{-0.02}^{+0.05}$ \\
\hline feat & $E W(\mathrm{eV})$ & $\begin{array}{l}<0.05 \\
-28.1_{-30.8}^{+28.1}\end{array}$ & $\begin{array}{l}<0.05^{+5.1} \\
-21.4_{-6.7}^{+5}\end{array}$ & $\begin{array}{l}<0.05 \\
-64.0_{-35.6}^{+33.8}\end{array}$ & $\begin{array}{l}<0.04 \\
-29.4_{-6.4}^{+8.1}\end{array}$ & $\begin{array}{l}<0.05 \\
-73.3_{-30.7}^{+29.6}\end{array}$ & $\begin{array}{l}<0.05 \\
-29.3_{-8.6}^{+3.6}\end{array}$ \\
\hline Fexxvi $\mathrm{K} \beta$ abs & $E_{\text {line }}(\mathrm{keV})$ & 8.26 fixed & $8.20_{-0.03}^{+0.07}$ & 8.26 fixed & $8.25_{-0.03}^{+0.05}$ & $8.11_{-0.01}^{+0.06}$ & $8.23_{-0.03}^{+0.07}$ \\
\hline line & $\begin{array}{l}\sigma(\mathrm{keV}) \\
E W(\mathrm{eV})\end{array}$ & $\begin{array}{l}<0.05 \\
>-41.1\end{array}$ & $\begin{array}{l}<0.05^{+6.0} \\
-25.0_{-7.0}^{+6.0}\end{array}$ & $\begin{array}{l}<0.05^{+15.7} \\
-15.7_{-56.6}^{+1}\end{array}$ & $\begin{array}{l}<0.05 \\
-27.3_{-9.6}^{+8.4}\end{array}$ & $\begin{array}{l}<0.05^{+36.5} \\
-69.1_{-37.9}^{+3}\end{array}$ & $\begin{array}{l}<0.06 \\
-19.3_{-10.4}^{+6.9}\end{array}$ \\
\hline Fe Xxv edge & $\begin{array}{l}E_{\text {line }}(\mathrm{keV}) \\
\tau\end{array}$ & $\begin{array}{l}8.83 \text { fixed } \\
<0.40\end{array}$ & $\begin{array}{l}8.83 \text { fixed } \\
0.19_{-0.02}^{+0.02}\end{array}$ & $\begin{array}{l}8.83 \text { fixed } \\
<0.42\end{array}$ & $\begin{array}{l}8.83 \text { fixed } \\
0.14_{-0.08}^{+0.05}\end{array}$ & $\begin{array}{l}8.83 \text { fixed } \\
<0.32\end{array}$ & $\begin{array}{l}8.83 \text { fixed } \\
0.28_{-0.02}^{+0.04}\end{array}$ \\
\hline Fexxvi edge & $\begin{array}{l}E_{\text {line }}(\mathrm{keV}) \\
\tau\end{array}$ & $\begin{array}{l}9.28 \text { fixed } \\
<0.22\end{array}$ & $\begin{array}{l}9.28 \text { fixed } \\
<0.04\end{array}$ & $\begin{array}{l}9.28 \text { fixed } \\
<0.50\end{array}$ & $\begin{array}{l}9.28 \text { fixed } \\
<0.03\end{array}$ & $\begin{array}{l}9.28 \text { fixed } \\
<0.20\end{array}$ & $\begin{array}{l}9.28 \text { fixed } \\
<0.01\end{array}$ \\
\hline $\begin{array}{l}\text { Fe broad emission } \\
\text { line }\end{array}$ & $\begin{array}{l}E_{\text {line }}(\mathrm{keV}) \\
\sigma(\mathrm{keV}) \\
E W(\mathrm{eV})\end{array}$ & $\begin{array}{l}6.24_{-0.24}^{+0.27} \\
0.8^{ \pm} 0.2 \\
260_{-160}^{+270}\end{array}$ & $\begin{array}{l}6.80_{-0.09}^{+0.13} \\
0.37_{-0.11}^{+0.29} \\
91_{-30}^{+9}\end{array}$ & $\begin{array}{l}6.80 \text { fixed } \\
0.3 \text { fixed } \\
<130\end{array}$ & $\begin{array}{l}6.85_{-0.05}^{+0.11} \\
0.35_{-0.14}^{+0.14} \\
96_{-18}^{+27}\end{array}$ & $\begin{array}{l}6.80 \text { fixed } \\
0.3 \text { fixed } \\
170_{-70}^{+90}\end{array}$ & $\begin{array}{l}6.77_{-0.09}^{+0.02} \\
0.26_{-0.04}^{+0.13} \\
141_{-40}^{+30}\end{array}$ \\
\hline
\end{tabular}

Examination of the fit residuals shows that, especially in the $3 \mathrm{PN}$ spectra, broad positive residuals are present near $6.7 \mathrm{keV}$, requiring the addition of a broad Gaussian line in all 3 fits with $E W$ s of $\sim 100 \mathrm{eV}$ and widths, $\sigma$, in the range $\sim 200-400 \mathrm{eV}$. The continuum parameters derived from all spectra are similar. For the multicolor disk-blackbody component $k T$ is in the range $0.5-0.7 \mathrm{keV}$ while the $k T$ of the blackbody is $1.1-1.2 \mathrm{keV}$. Both components are absorbed by an $N_{\mathrm{H}}$, of $3.1-3.8 \times 10^{22}$ atom $\mathrm{cm}^{-2}$. Without the addition of the emission and absorption features discussed below the fit quality is rather poor with a $\chi_{\nu}^{2}$ of 3.5-4.6. The addition of emission and absorption lines (modeled by Gaussians) successively decreases the $\chi_{\nu}^{2}$ to 2.0-2.4 (see below). The spectral parameters for the multicolor disk-blackbody and blackbody are consistent with those obtained from the ASCA observation by Asai et al. (2000).

Two strong absorption features are observed in all 6 spectra at around 6.7 and $7.0 \mathrm{keV}$ (see Table 2). These features are consistent with being due to resonant $K$ shell absorption from highly ionized iron Fe XXV and Fe XXVI, respectively. The deeper $7.0 \mathrm{keV}$ feature is almost certainly the absorption feature observed from GX $13+1$ by Ueda et al. (2001) using ASCA. The addition of both features is highly significant. Including the $7.0 \mathrm{keV}$ line reduces the $\chi_{\nu}^{2}$ by $0.6-0.8$ while the addition of the $6.7 \mathrm{keV}$ feature reduces the $\chi_{\nu}^{2}$ by $0.3-0.6$. Two other absorption features at around 7.8 and $8.2 \mathrm{keV}$ are also detected here for the first time in GX $13+1$ in all the PN spectra, while in the MOS1 spectra they are visible only in one observation (Obs. ID 1001), consistent with the lower effective area of this instrument, compared to the PN. The addition of these features reduces the $\chi_{\nu}^{2}$ by $0.15-0.3$ and 0.04-0.07 for the 7.8 and $8.2 \mathrm{keV}$ features, respectively. In both the MOS1 and PN spectra an absorption feature at $\sim 4.1 \mathrm{keV}$ is also evident. This is consistent with being due to resonant $\mathrm{K} \alpha$ absorption from $\mathrm{H}$-like calcium (CaXx). All the detected absorption lines and their parameters are listed in Table 2. We note that there is a strong interplay between the parameters of the broad emission line and the $E W \mathrm{~s}$ of the two strong absorption features since these features cut strongly into the emission line. This is most clearly seen in Fig. 1 which shows the best-fit PN spectra together with expanded plots of the spectral regions that include the Fe features.

Line energies measured using the PN Timing Mode spectra were expected to be slightly higher than their "true" values due to uncertainties in the calibration of Timing Mode. However, a comparison of the energies derived from the MOS1 and PN spectra for the two strongest absorption lines (Fe XXVI and Fe XXV K $\alpha$ ) shows that they are consistent at $90 \%$ confidence. We therefore present in Table 2 the PN energies without any adjustment. The identification of the fainter absorption features at 7.8 and $8.2 \mathrm{keV}$, is uncertain. They may be tentatively identified with Fexxv (7.88 keV) and Fexxvi $K \beta(8.26 \mathrm{keV})$, or with NixxVII $(7.80 \mathrm{keV})$ and NixxVIII $K \alpha(8.10 \mathrm{keV})$, respectively (e.g., Kotani et al. 2000). 
Close inspection of the residual plots (Fig. 1) reveals the presence of an emission feature near $8.5 \mathrm{keV}$. A fit to this feature with a Gaussian model gives an energy of $8.51 \pm 0.05 \mathrm{keV}$ and an $E W$ in the range $25-35 \mathrm{eV}$. Since this energy is close to those of known PN background features due to $\mathrm{Zn} K \alpha$ and $\mathrm{Cu} K \beta$ (an even stronger line is present in the background spectrum near $8.05 \mathrm{keV}$ from $\mathrm{Cu} K \alpha$ ) we investigated whether this feature could originate from the background. In the PN Timing Mode, data from the on-axis CCD are continually clocked across the target region and data are extracted with only one dimension of spatial information. We searched all the publically available XMM-Newton observations but were unable to find any blank field Timing Mode data from which to extract a background spectrum. Since the internal background is spatially very structured, particularly in the $K$ - fluorescent lines of metal components of the camera body, we constructed a background spectrum from the identical region covered by Timing Mode from Imaging Mode data, taking care to apply the appropriate exposure time correction. From this spectrum we find that the observed $8.5 \mathrm{keV}$ feature is a factor 100 more intense than the background features. We therefore conclude that the observed feature is not a background artifact. The energy of the emission feature does not correspond to any prominent emission line from an abundant element. However, it is just below that of the He-like Fe edge at $8.83 \mathrm{keV}$. We therefore attempted to model this feature as an absorption edge. The inclusion of an edge at $8.83 \mathrm{keV}$ accounts for the excess around $8.5 \mathrm{keV}$ and decreases the $\chi_{\nu}^{2}$ to $1.6-1.7$. The best-fit parameters for all the discrete features can be found in Table 2, where the upper limits to the presence of a H-like Fe edge (at $9.28 \mathrm{keV}$ ) are also reported. A narrow emission feature near $6.4 \mathrm{keV}$ was reported by Ueda et al. (2001). Addition of this narrow feature with an energy close to $6.4 \mathrm{keV}$ resulted in a decrease of the $\chi^{2}$ of $4-7$, which is not significant.

We note that the parameters derived for the broad emission feature from the PN and MOS1 are inconsistent. The energy derived from the MOS is lower, while the width of the line is significantly broader. This is not caused by the fitting method (e.g. a local minimum) since the addition of the line with the $\mathrm{PN}$-derived parameters to the MOS spectral model results in a clearly worse $\chi^{2}$ $\left(\Delta \chi^{2}>100\right)$, and the difference between the model and the MOS data is up to $10-15 \%$, i.e. larger than the calibration uncertainty. Since a large fraction of the line emission is removed by the narrow absorption components it is difficult to reliably constrain the emission component. We therefore await an improved EPIC calibration before the parameters of the broad emission line can be accurately determined.

\section{Discussion}

There is a growing evidence that narrow absorption lines from highly ionized material are a common feature of LMXRB spectra. They were first detected from the superluminal jet sources GRO J1655-40 (Ueda et al. 1998; Yamaoka et al. 2001) and GRS 1915+105 (Kotani et al. 2000; Lee et al. 2001) with ASCA. Absorption lines due to Fe XXV and Fe XXVI were observed from GRO J1655-40 and did not show any obvious dependence of their $E W_{\mathrm{s}}$ on orbital phase. Both X-ray and optical observations indicate that GRO J1655-40 is most probably a binary system viewed at a high inclination angle of $\sim 70^{\circ}$ (Kuulkers et al. 1998; van der Hooft et al. 1997; Greene et al. 2001). ASCA observations of GRS 1915+105 revealed, in addition, absorption features due to CaXx, NiXXVII and NiXXVIII. This source has been observed also with the Chandra HETGS which detected absorption edges of $\mathrm{Fe}, \mathrm{Si}, \mathrm{Mg}$, and $\mathrm{S}$ as well as resonant absorption features from Fe XXV and Fe XXVI and possibly Ca XX (Lee et al. 2001). The recent discovery of similar narrow iron absorption features in X-ray binaries containing neutron stars (GX 13+1, Ueda et al. 2001; MXB 1658-298, Sidoli et al. 2001 and X 1624-490, Parmar et al. 2002), rules out the possibility that such features are peculiar to superluminal jet sources and related in some way to the jet formation mechanism. Chandra HETGS observations have also convincingly detected narrow absorption features due to Ne IX and Fe XVIII from the transient black hole candidate XTE J1650-500 (Miller et al. 2002). These lines are may be due to absorption in an ionized accretion disk atmosphere or wind.

The XMM-Newton observations reported here reveal a much more complex range of emission and absorption components from GX $13+1$ than even the ASCA analysis of Ueda et al. (2001) had found. In addition to the Fe XXVI K $\alpha$ absorption feature additional narrow absorption features which may be identified with $\mathrm{Ca} \mathrm{Xx}$ and Fe XXv K $\alpha$ and with Fe XXV and Fe Xxvi K $\beta$ are evident in the XMM-Newton spectra. In addition a deep edge at $8.83 \mathrm{keV}$ is clearly required. We note that the feature at $7.61 \pm 0.13 \mathrm{keV}$ which Ueda et al. (2001) model as an edge is more clearly seen to be a line absorption feature in the XMM-Newton PN spectra (which we identify with $\mathrm{Fe} \operatorname{xxV} \mathrm{K} \beta$ ). It is interesting to speculate as to why a deep $(\tau \sim 0.2)$ edge from Fe XXV is evident whereas the PN upper-limits to the optical depth for an Fe XXVI edge at $9.28 \mathrm{keV}$ of $\lesssim 0.04$ exclude a similar feature. This is in contrast to the corresponding absorption lines, where the $E W$ of the Fe XXVI transitions exceeds that of Fe XXV in all the spectra.

In order to convert the observed $E W$ s of the lines into ion column densities, we assumed the curve of growth analysis performed by Kotani et al. (2000). In this way, the column densities of the lines can be estimated for a range of kinematic temperatures of the absorbing material, where this temperature includes contributions from thermal motions as well as any bulk motions or turbulence. An Fe abundance of $4.7 \times 10^{-5}$ was assumed (Anders $\&$ Grevesse 1989). The column densities for Fexxv and Fexxvi, calculated for a range of assumed temperatures $(0.1,1,10,100$ and $1000 \mathrm{keV})$, are shown in Fig. 2 for only one observation, for clarity. Indeed, similar $E W$ s have 


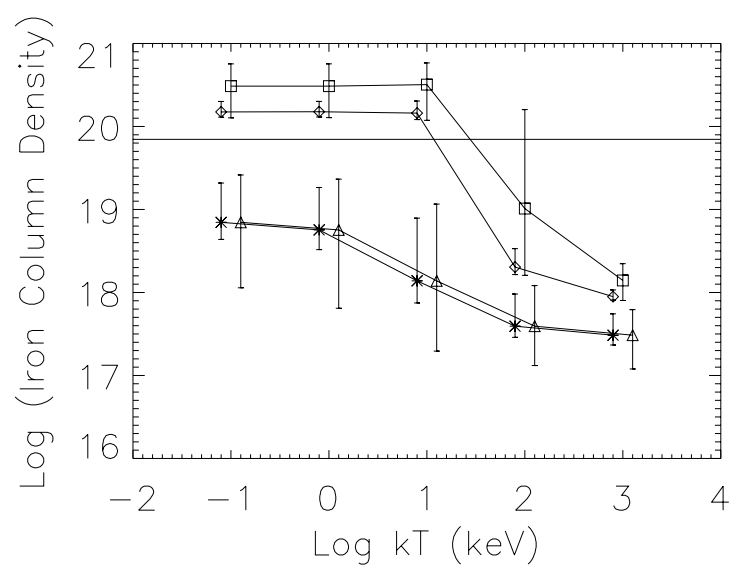

Fig. 2. Fe column densities for the two $\mathrm{K} \alpha$ absorption lines from the first (ID. 0101) MOS1 (squares are for Fe XxVI, triangles for Fexxv) and PN observations (diamonds are for Fe XXvi, stars for Fexxv). For the other two observations (ID. 0901 and ID. 1001) similar results have been obtained. The horizontal line marks the upper limit to the Fe column density corresponding to a Thomson optical depth equal to unity. A small horizontal shift has been applied for clarity. Column densities are in units of $\mathrm{cm}^{-2}$.

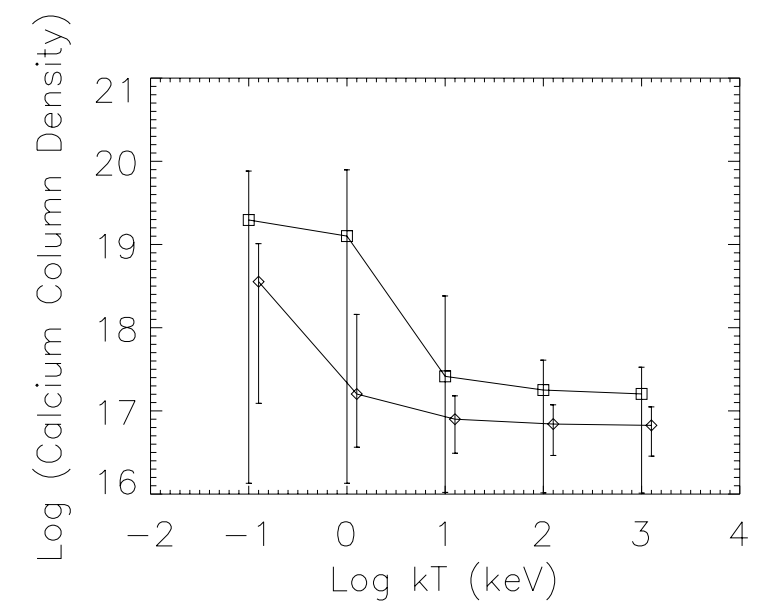

Fig. 3. Results of the curve of growth analysis for the CaXx line, using only results from the first observation (Obs. ID 0101). Similar values are obtained from the other two observations. Squares corresponds to PN results, diamonds to MOS1. A small horizontal shift has been applied for clarity. Column densities are in units of $\mathrm{cm}^{-2}$.

been measured in all the three observations. A lower kinematic temperature requires a higher ion column density, and hence a higher $N_{\mathrm{H}}$. A constraint on the absorbing Fe column density can be derived from the fact that the plasma should be optically thin to Thomson scattering $\left(N_{\mathrm{H}}<1.5 \times 10^{24}\right.$ atom $\left.\mathrm{cm}^{-2}\right)$, otherwise the absorption lines would be strongly diminished. Assuming a solar Fe abundance, implies an Fe column density $<7 \times 10^{19} \mathrm{~cm}^{-2}$, which translates into a temperature $\gtrsim 10 \mathrm{keV}$. The upper limits to the velocity widths of the two narrow lines of $<1800 \mathrm{~km} \mathrm{~s}^{-1}$ (Fe XXV) and $<1900 \mathrm{~km} \mathrm{~s}^{-1}$ (Fe XXVI), provide not very constraining upper limits to the $k T$ of 600 $650 \mathrm{keV}$. The $E W$ s of the highly ionized Fe K lines (and thus the ions column densities) are similar to those measured in the eclipsing, dipping LMXRB MXB 1658-298 (Sidoli et al. 2001) with XMM-Newton, and a factor $\sim 3$ higher than that observed in the LMXRB X 1624-490 (Parmar et al. 2002). The results of the curve of growth analysis for the Caxx line are shown in Fig. 3. The ionized calcium column density ranges from $10^{16} \mathrm{~cm}^{-2}$ to $10^{20} \mathrm{~cm}^{-2}$, depending on the kinematic temperature. No useful constraints come from the upper limit to the velocity width of $<3700 \mathrm{~km} \mathrm{~s}^{-1}$, that translates into a temperature of $\lesssim 1900 \mathrm{keV}$.

The line centroids of the Fe Xxv and Fe Xxvi K $\alpha$ do not show evidence for any velocity shifts, except in the second observation (Obs. ID 0901) where blue-shifts of $-2700 \pm$ $2200 \mathrm{~km} \mathrm{~s}^{-1}\left(\right.$ FexxV K $\alpha$ ) and $-3900 \pm 1700 \mathrm{kms}^{-1}$ (Fexxvi $\mathrm{K} \alpha$ ) are evident. In this same observation, a blue-shift in the CaXx feature of $-5100 \pm 2200 \mathrm{~km} \mathrm{~s}^{-1}$ is also detected. For most observations, the MOS energy calibration is correct to within $\sim 5 \mathrm{eV}$, or a velocity shift of $\sim 220 \mathrm{kms}^{-1}$ at $7 \mathrm{keV}$ and $\sim 400 \mathrm{~km} \mathrm{~s}^{-1}$ at $4 \mathrm{keV}$. Individual observations have been analysed where the Mn K $\alpha$ internal calibration line appears to be discrepant by $\sim 30 \mathrm{eV}$, but these are all associated with anomalous instrument temperatures resulting from spacecraft irregularities. Such conditions did not pertain for the observations reported here. The absorption lines at higher energy cannot be unambiguously identified. These features are only clearly detected in the MOS1 in the third observation (Obs. ID. 1001). The measured line energy of $7.84 \pm 0.04 \mathrm{keV}$ can be associated with both the Fe XxV K $\beta$ (at $7.88 \mathrm{keV}$ ) and the Ni XXVII K $\alpha$ (at $7.80 \mathrm{keV}$ ) feature. The absorption line at $\sim 8.1 \mathrm{keV}$ can be similarly associated with either an Fe xxvi K $\beta$ transition (at $8.212 \mathrm{keV}$ ) or with a NixxvIII K $\alpha$ feature at $8.034 \mathrm{keV}$.

Since the orbital period of GX $13+1$ has not been confirmed, we cannot reliably estimate a geometry and size for the absorbing plasma around the central $\mathrm{X}$-ray source. If the 25 day period is confirmed to be orbital, we note that the XMM-Newton observations reported here only cover a small fraction $(\sim 10 \%)$ of an orbital cycle. All we can say is that all the narrow absorption lines are consistent with being present with the same $E W$ in all analyzed spectra. This implies that the absorbing plasma is stable in the line of sight over timescales of few days suggesting a stable geometry, as in the case of MXB 1658-298 and X 1624-490, and excluding the possibility of absorbing clouds temporarily passing in front of the central source producing the absorption. Additionally, the fact that the $E W$ s of the Fe xxvi line measured here are consistent with that observed with ASCA (Ueda et al. 2001) a few years ago, again supports this conclusion.

Acknowledgements. Based on observations obtained with XMM-Newton, an ESA science mission with instruments and contributions directly funded by ESA member states and the USA (NASA). We thank T. Kotani for making his curve of growth software available. 


\section{References}

Anders, E., \& Grevesse, N. 1989, Geochim. Cosmochim. Acta, 53,197

Asai, K., Dotani, T., Nagase, F., \& Mitsuda, K. 2000, ApJS, 131,571

Bandyopadhyay, R. M., Shahbaz, T., Charles, P. A., \& Naylor, T. 1999, MNRAS, 306, 417

Bandyopadhyay, R. M., Charles, P. A., Shahbaz, T., \& Wagner, R. M. 2002, ApJ, in press

Charles, P. A., \& Naylor, T. 1992, MNRAS, 255, 6

Christian, D. J., \& Swank, J. H. 1997, APJS, 109, 177

Corbet, R. 1996, IAUC., 6508

Cottam, J., Kahn, S. M., Brinkman, A. C., den Herder, J. W., \& Erd, C. 2000, A\&A, 365, L277

Fender, R. P., \& Hendry, M. A. 2000, MNRAS, 317, 1

Fleischman, J. R. 1985, A\&A, 153, 106

Greene, J. Bailyn, . D., \& Orosz, J. A. 2001, ApJ, 554, 1290

Grindlay, J. E., \& Seaquist, E. R. 1986, ApJ, 310, 172

Homan, J., van der Klis, M., Wijnands, R., et al. 1998, ApJ, 499, L41

Jansen, F., Lumb, D., Altieri, B., et al. 2001, A\&A, 365, L1

Kotani, T., Ebisawa, K., Dotani, T., et al. 2000, ApJ, 539, 413

Kuulkers, E., Wijnands, R., Belloni, T., et al. 1998, ApJ, 494, 753
Lee, J. C., Schulz, N. S., Reynolds, C. S., Fabian, A. C., \& Blackman, E. G. 2001, Proc. of X-ray Astronomy 2000, [astro-ph/0012111]

Matsuba, E., Dotani, T., Mitsuda, K., et al. 1995, PASJ, 47, 575

Miller, J., Wijnands, R., Wojdowski, P., et al. 2002, ATel, 81

Mitsuda, K., Inoue, H., Koyama, K., et al. 1984, PASJ, 36, 741

Morrison, D., \& McCammon, D. 1983, ApJ, 270, 119

Naylor, T., 1991, Charles, P. A., \& Longmore, A. J. 1991, MNRAS, 252, 203

Parmar, A. N., Oosterbroek, T., Boirin, L., \& Lumb, D. 2002, A\&A, in press

Sidoli, L., Oosterbroek, T., Parmar, A. N., et al. 2001, A\&A, 379,540

Schulz, N. S., Hasinger, G., \& Trumper, J. 1989, A\&A, 225, 48

Strüder, L., Briel, U., Dennerl, K., et al. 2001, A\&A, 365, L18

Turner, M. J. L., Abbey, A., Arnaud, M., et al. 2001, A\&A, $365, \mathrm{~L} 27$

Ueda, Y., Inoue, H., Tanaka, Y., et al. 1998, ApJ, 492, 782

Ueda, Y., Asai, K., Yamaoka, K., Dotani, T., \& Inoue, H. 2001, ApJ, 556, L87

Van der Hooft, F., Groot, P. J., Shahbaz, T., et al. 1997, MNRAS, 286, L43

Yamaoka, K., Ueda, Y., Inoue, H., et al. 2001, PASJ, 53, 179 